\title{
Molecular Characterization of Glucose-6-Phosphate Dehydrogenase Deficiency in Abu Dhabi District, United Arab Emirates
}

\section{Saleh Ahmed Bin Amro ${ }^{1,2 *}$, Eiman Al Zaabi², Shakir Hussain ${ }^{2}$, Amira Mahmoud Aly $^{2}$, Huda Salman Baqir ${ }^{1}$, Abdul-Hamid Ahmed Zaki ${ }^{1}$, Sultan Ayesh Mohammed Saghir ${ }^{3}$ and Narazah Mohammed Yusoff ${ }^{1}$}

${ }^{1}$ Cluster of Regenerative Medicine, Advanced Medical and Dental Institute (AMDI), Universiti Sains Malaysia (USM), 11800 Penang, Malaysia, ${ }^{2}$ Hematopathology Section, Department of Pathology and Laboratory Medicine, Mafraq Hospital, Abu Dhabi, United Arab Emirates (UAE), ${ }^{3}$ School of Pharmacy, Pharmacology Department, University Sains Malaysia (USM), Penang, Malaysia

${ }^{*}$ For correspondence: Email: abuatheer1@yahoo.com; Tel: +971-2-5011317, +971-552987641/507528850; Fax: +971-25011682

\begin{abstract}
Purpose: To investigate the frequency of glucose-6-phosphate dehydrogenase (G6PD) variants and their associated enzyme deficiencies among different age groups of individuals in Abu Dhabi, United Arab Emirates (UAE).

Methods: A total of 15,995 patients (6302 UAE nationals and 9693 non-UAE nationals) who presented at Mafraq Hospital, Abu Dhabi, UAE between January 2006 and January 2009 were screened for G6PD deficiency using fluorescent spot test. Molecular analysis including polymerase chain reactionrestriction fragment length polymorphism (PCR-RFLP), denaturing high performance liquid chromatography (DHPLC) and DNA sequencing were utilized to identify common mutations in individuals with G6PD deficiency.

Results: The prevalence of G6PD deficiency among UAE nationals was $7.4 \%$ and non-UAE nationals $3.8 \%$. UAE males showed prevalence of $11.6 \%$ while for UAE females it was $3.6 \%$. The prevalence of G6PD deficiency among non-UAE nationals was 5 and $1.7 \%$ for males and females, respectively. The Mediterranean mutation, $563 C \rightarrow T$, was predominant in non-UAE nationals.

Conclusion: G6PD Mediterranean mutation is the most prevalent mutation underlying G6PD deficiency followed by Aures mutations in both UAE nationals and non-UAE nationals. On the other hand, Africa $A^{-}$ was found to be more in non-UAE compared with UAE nationals.
\end{abstract}

Keywords: Glucose-6-Phosphate Dehydrogenase deficiency, Mutation, Abu Dhabi, Polymerase chain reaction-restriction, Fragment length polymorphism

Tropical Journal of Pharmaceutical Research is indexed by Science Citation Index (SciSearch), Scopus, International Pharmaceutical Abstract, Chemical Abstracts, Embase, Index Copernicus, EBSCO, African Index Medicus, JournalSeek, Journal Citation Reports/Science Edition, Directory of Open Access Journals (DOAJ), African Journal Online, Bioline International, Open-J-Gate and Pharmacy Abstracts

\section{INTRODUCTION}

Glucose-6-phosphate dehydrogenase deficiency (G6PD) is an essential enzyme that reduces nicotinamide adenine dinucleotide phosphate (NADP) to the reduced form (NADPH). G6PD deficiency is the most common human enzymopathy that causes a spectrum of diseases including acute and chronic hemolysis of red blood cells, hereditary non spherocytic hemolytic anemia and life threatening kernicterus in newborn. In this condition, acute hemolysis is 
triggered by infections, favism (fava beans ingestion) and drugs [1].

This X-linked inherited disorder most commonly affects individuals of African, Asian, Mediterranean, and Middle-East regions. The G6PD gene is $20 \mathrm{~kb}$ in length and situated on the Xq28 region of $X$ chromosome and comprising 13 exons and 12 introns. The clinical symptoms of the disease are usually confined to hemizygous male, although female carriers sometimes also suffer [2]. Different levels of enzyme deficiency appear due to different gene mutations. About 140 different G6PD mutations have been reported, almost all of which are with single base mutations resulting in replacement of amino acids [2]. In an earlier study performed in the Central Hospital, Abu Dhabi, prevalence of G6PD deficiency in UAE nationals was reported to be more than $25 \%$ [3].

G6PD deficiencies have been reported in Omani (26\%), Bahraini (21\%) and Baluchi (28 to $45 \%$ ) populations [4]. Previous studies illustrated that the frequency of G6PD deficiency in UAE males ranges from 11 to $15 \%$, whereas it was $5 \%$ for UAE females [5]. This study aims to determine the prevalence of G6PD deficiency and to characterize its molecular basis in a large sample of UAE and non-UAE nationals residing in $\mathrm{Abu}$ Dhabi using fluorescent spot test, PCR-RFLP, DHPLC and DNA sequencing.

\section{EXPERIMENTAL}

\section{Subjects}

This study was a cross-sectional investigation, in which 15,995 subjects including UAE as well as non-UAE nationals participated. All patients, children and adults inclusive, who presented in Mafraq Hospital for medical treatment and newborns at Mafraq Hospital from January 2006 to January 2009, were investigated for G6PD deficiency.

This study was conducted in a total of 15995 subjects. Of these, $8984(56.2 \%)$ were males and $7011(43.8 \%)$ females. Of the study population, 6302 subjects were UAE nationals (2996 males and 3306 females) while 9693 were non-nationals (5988 males and 3705 females).

Screening for G6PD deficiency was done by performing fluorescent spot test along with routine lab tests. Peripheral blood samples were collected in EDTA tubes for complete blood count (CBC), reticulocytes count, blood film, and qualitative florescent spot test. Hematological analysis were performed using Sysmex XE 2100 which. Bilirubin, Glutamic-pyruvic transaminase (GPT), glutamic-oxaloacetic transaminase (GOT), alkaline phosphatase (ALP) and lactate dehydrogenase (LDH) were measured on Roche - Hitachi Cobas 6000. positive G6PD deficient subjects $t$ were further evaluated for quantitative G6PD assay, PCR-RFLP, DHPLC and DNA sequencing.

\section{Qualitative and quantitative assays for G6PD}

For qualitative determination, spot screening test was used to detect the fluorescence of NADPH at $365 \mathrm{~nm}$ UV light in complete darkness. Reduction of NADP- to NADPH occurred in the presence of G6PD, where G6PD activity was calculated and expressed as $\mathrm{IU} / \mathrm{g}$ haemoglobin (Hb).

Quantitative determination was carried out using commercially available kits (Pointe Scientific Inc, Canton 48188, MI, USA) by measuring the rate of absorbance change at $340 \mathrm{~nm}$ due to the reduction of NADP to NADPH when a sample was incubated with $\mathrm{G} 6 \mathrm{P}$ at $37^{\circ} \mathrm{C}$ for $10 \mathrm{~min}$.

\section{Molecular studies}

DNA was extracted from G6PD-deficient blood using kit of Qiagen Company, Germany. Purified DNA was solubilized in a TE buffer (10 mM Tris$\mathrm{HCL}, 1 \mathrm{mM}$ EDTA, pH 8.0) and stored at $-20{ }^{\circ} \mathrm{C}$. The DNA of all samples were subjected to PCRRFLP to detect Mediterranean (563C-T) mutation, African $A^{-}$(202G-A), Aures143T-C, Chatham 1003G-A, and silent polymorphism mutation 1311C-T according to previously described protocols [2]. The PCR reaction was carried out for detection of Mediterranean mutation using one pair of oligonucleotides as follows: Forward primer B 5' ACTCCCGAAGAG GGGTTCAAGG $3^{\prime}$ and reverse primer $\mathrm{J} 5^{\prime}$ CCAGCCTCCCAGGAGAGAGGAAG3'. The PCR reaction to amplify exons 6 and 7 was initiated by denaturation at $94{ }^{\circ} \mathrm{C}$ for $5 \mathrm{~min}$ followed by 30 cycles of denaturation at $94{ }^{\circ} \mathrm{C}$ for 45 seconds, annealing at $56{ }^{\circ} \mathrm{C}$ for 45 seconds, and extension at $72{ }^{\circ} \mathrm{C}$ for $45 \mathrm{~s}$ and final extension at $72{ }^{\circ} \mathrm{C}$ for $5 \mathrm{~min}$. Amplification was done using one unit of Taq DNA polymerase per $\mu \mathrm{L}$ in a final volume of $25 \mu \mathrm{L}$. DNA amplification was done using five sets of primers covering from third exon to the eleventh exon as described previously [6].

The six restriction enzymes used for detection of mutations were as follows: Mboll for Mediterranean mutation, Mbol for Aures mutation, Hps92II for African A- mutation, BstXI for Chatham mutation and $B c / l$ for silent 1311 
polymorphism mutation. DNA sequences were determined using forward and reverse primers. $\mathrm{ABI}$ prism Biosystem BigDye terminator chemistry was used for sequence detections on an $\mathrm{ABI} 3730 \mathrm{XL}$ capillary-based DNA sequence after the identification of mutation through RFLPPCR. The PCR amplification of the entire coding sequence of G6PD gene was performed using eleven sets of primers for South East Asia (SEA) mutations prepared from Transgenomic Company using Primer Extension (PE) analysed by DHPLC.

DHPLC analysis was conducted to determine eleven SEA mutations which are as follows: $95 \mathrm{~A} \rightarrow \mathrm{G}, \quad 392 \mathrm{G} \rightarrow \mathrm{T}, \quad$ 487G $\rightarrow \mathrm{A}, \quad$ 493A $\rightarrow \mathrm{G}$, $592 \mathrm{C} \rightarrow \mathrm{T}, \quad 871 \mathrm{G} \rightarrow \mathrm{A}, \quad 1024 \mathrm{C} \rightarrow \mathrm{T}, \quad 1360 \mathrm{C} \rightarrow \mathrm{T}$, $1376 \mathrm{G} \rightarrow \mathrm{T}, 1388 \rightarrow \mathrm{GA}$ and polymorphism silent mutation $1311 \mathrm{C} \rightarrow \mathrm{T}$.

\section{Ethical issues}

The study was approved by the Research Ethics Committee of the Mafraq Hospital, Abu Dhabi, UAE. Informed consents were obtained from the patients or their legal guardians before taking samples.

\section{Statistical analysis}

Statistical Package for Social Sciences (SPSS) version 16 was used to analyse the results of this study using independent- samples $t$ - test. Differences between normal and G6PD-deficient subjects were considered significant at $P<0.05$.

\section{RESULTS}

\section{Prevalence of G6PD deficiency}

This study considered one of the few reports which have been done in Abu Dhabi District to evaluate the frequency of different G6PD mutations. The prevalence of G6PD-deficient individuals among the total subjects (UAE nationals and Non-UAE nationals) in Abu Dhabi District was $5.2 \%$ (830 out of 15995). Males were $7.2 \%$ (648 out of 8984 ) compared to $2.6 \%$ (182 out of 7011) for females. In addition, the difference in prevalence of G6PD deficiency between male and female were statistically significant $(P<0.001)$, indicating that there might a link between gender and G6PD deficiency. Among UAE nationals in the Abu Dhabi district, the G6PD deficiency was tested in 6302 subjects (2996 males and 3306 females), 7.4\% were found to be deficient. Males constituted $11.6 \%$ compared to $3.6 \%$ for females. The prevalence of G6PD deficiency among non-UAE nationals was $3.8 \%$. Deficient non-UAE males were $5 \%$ compared to $1.7 \%$ for females (Table 1 ).

\section{Prevalence of G6PD mutations}

The distribution of mutations in the Abu Dhabi districts was obtained from a total of 224 G6PD deficient subjects. Six mutations were detected. The first most common mutation assessed was G6PD Mediterranean (563C-T) (Figure 1). Out of the 224 subjects, $173(77.2 \%)$ had G6PD Mediterranean, 27 (12\%) were G6PD Aures (143T-C), 21 (9.4\%) G6PD manifested Africa A(202 G-A), 1 case (0.5\%) had G6PD Chatham (1003G-A) while 2 cases $(0.9 \%)$ of G6PD Union (1360C-T) seen were from Philippines.

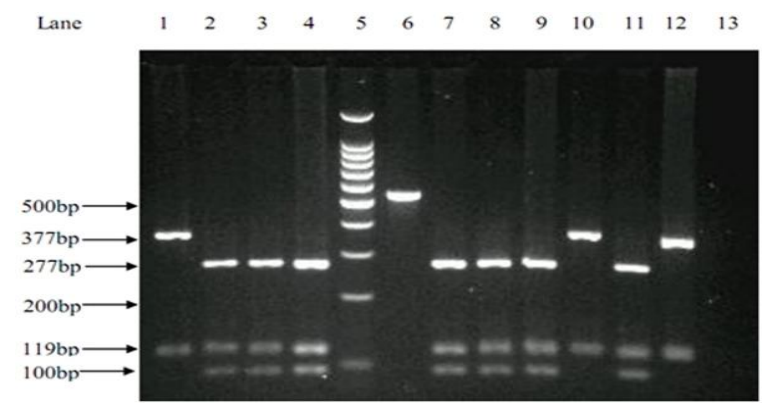

Figure 1: PCR products digested with Mboll restriction enzyme, analysis of the PCR product before and after digestion of Mboll enzyme for the detection of Mediterranean mutation in G6PD gene on $2 \%$ agarose gel from left to right. The arrow shows restriction sites in normal and deficient patients. Lanes 1, 10, and 12: normal samples; Lane 2: positive control, Lane 5: 100 bp ladder marker; Lanes 3, 4, 7, 8, 9, 11: G6PD Mediterranean mutation; Lane 6: PCR product, and Lane 13 free nuclease water (negative control).

\section{Prevalence of G6PD mutations among UAE and non-UAE nationals}

Table 2 shows the prevalence of G6PD mutation in UAE and non-UAE nationals. Seventy eight percent of UAE subjects had Mediterranean mutation $(563 \mathrm{C} \rightarrow \mathrm{T}) ; \quad 12 \%$ Aures mutation $(143 \mathrm{~T} \rightarrow \mathrm{C}) ; 8 \%$ Africa $\mathrm{A}^{-}$mutation $(202 \mathrm{G} \rightarrow \mathrm{A})$ while $1 \%$ manifested Chatham mutation $(1003 \mathrm{G} \rightarrow \mathrm{A})$. On the other hand, $74.1 \%$ of nonUAE national subjects had Mediterranean mutation $(563 \quad \mathrm{C} \rightarrow \mathrm{T})$; $12 \%$ Aures mutation $(143 \mathrm{~T} \rightarrow \mathrm{C}) ; 11.1 \%$ were Africa A-mutation (202 $\mathrm{G} \rightarrow \mathrm{A}$ ) and $2.5 \%$ Union mutation $(1360 \mathrm{C} \rightarrow \mathrm{T})$

None of the South-East Asia mutations was identified in any subject in our study except the two Filipino patients were positive for a union variant $1360 \mathrm{C} \rightarrow \mathrm{T}$ (Figure 2 ). The polymorphism silent mutation, $1311 \mathrm{C}-\mathrm{T}$, was observed in 170 out of the $173 \quad(98.3 \%)$ subjects with Mediterranean mutation (Figure 3). 
Table 1: Prevalence of G6PD deficiency by gender and nationality

\begin{tabular}{|c|c|c|c|c|c|c|c|}
\hline \multirow{2}{*}{ Parameter } & \multicolumn{2}{|c|}{ Total } & \multicolumn{2}{|c|}{ UAE nationals } & \multicolumn{2}{|c|}{ Non- UAE nationals } & \multirow[t]{2}{*}{ Total } \\
\hline & Male & Female & Male & Female & Male & Female & \\
\hline $\begin{array}{l}\text { Patients } \\
\text { (N) }\end{array}$ & 8984 & 7011 & 2996 & 3306 & 5988 & 3705 & 15995 \\
\hline Deficient & $648(7.2 \%)$ & $182(2.6 \%)$ & $347(11.6 \%)$ & $119(3.6 \%)$ & $301(5 \%)$ & $63(1.7 \%)$ & 830 \\
\hline $\begin{array}{l}\text { Total } \\
P \text {-value }\end{array}$ & \multicolumn{2}{|c|}{$830(5.2 \%)$} & \multicolumn{2}{|c|}{$466(7.4 \%)$} & \multicolumn{2}{|c|}{$364(3.8 \%)$} & \\
\hline
\end{tabular}

Table 2: Mutations in UAE and non-UAE nationals

\begin{tabular}{lcccccc}
\hline & & \multicolumn{3}{c}{ Mutation type } \\
\cline { 3 - 7 } Nationality & Number & Mediterranean & Aures & Africa202 & Chatham & Union \\
\hline UAE & 143 & $113(79 \%)$ & $17(11.9 \%)$ & $12(8.4 \%)$ & $1(0.7 \%)$ & 0 \\
Non-UAE & 81 & $60(74.1 \%)$ & $10(12.3 \%)$ & $9(11.1 \%)$ & 0 & $2(2.5 \%)$ \\
Total & 224 & $173(76.9 \%)$ & $27(12 \%)$ & $21(9.3 \%)$ & $1(0.4 \%)$ & $2(0.9 \%)$ \\
\hline
\end{tabular}

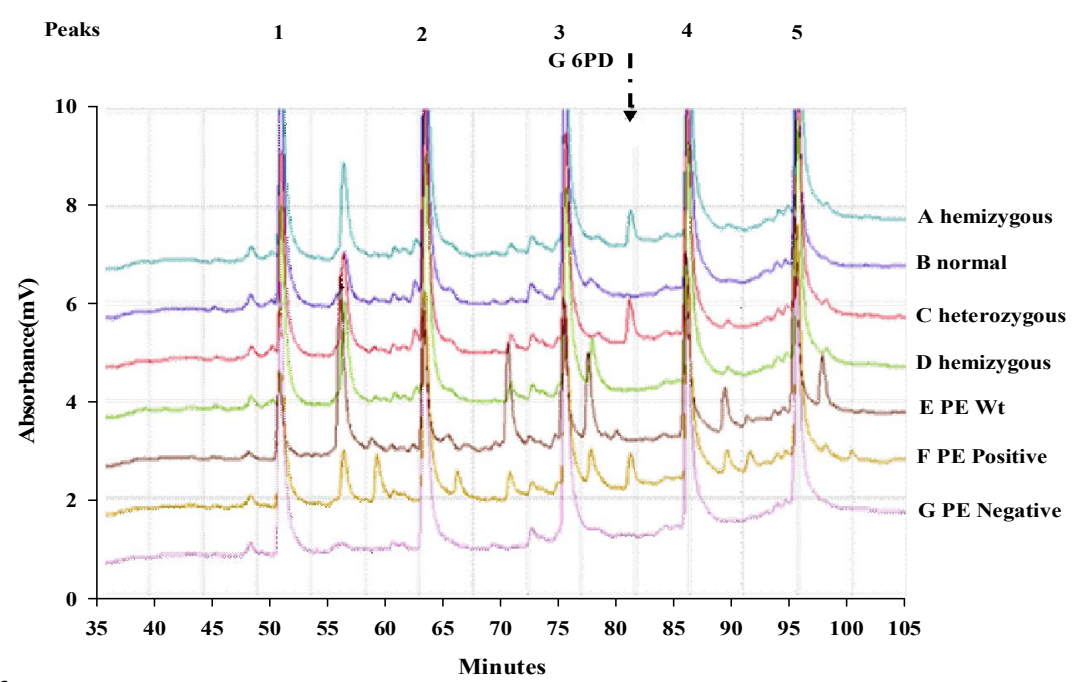

Figure 2: Detection of five South East Asia (SEA) mutations (group 2) using DHPLC assay, including positive, negative control, and wild type. The peaks are 1, 2, 3, 4, and 5 that correspond to 487A, 493G, 1360T, 871A, and1024T, respectively. Only two patients carrying the 1360T mutation appeared with additional peak.

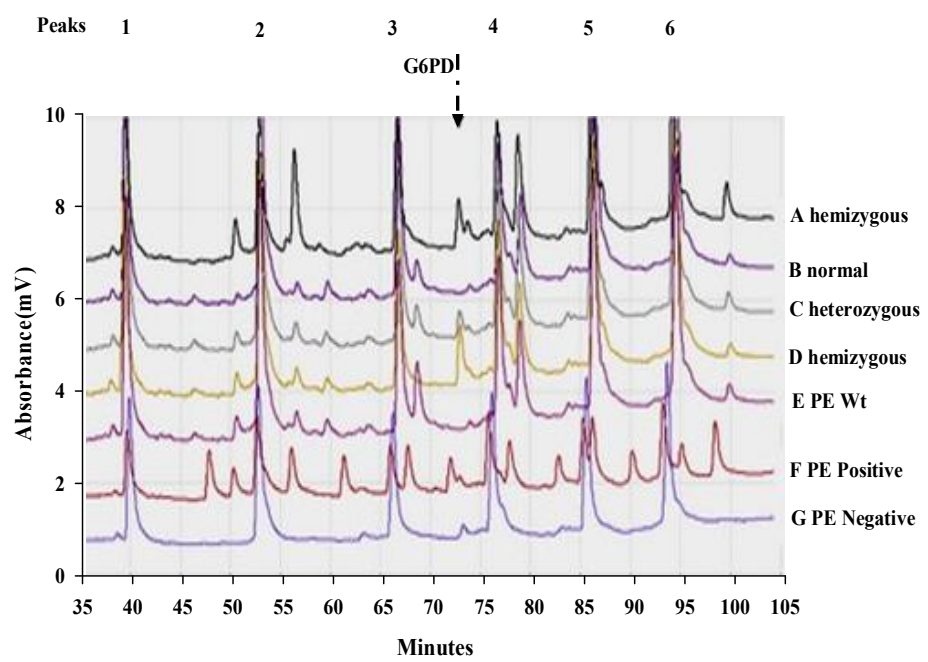

Figure 3: Detection of six South East Asia (SEA) mutations (group 1) using DHPLC assay, including positive, negative control, and wild type. The peaks are 1, 2, 3, 4, 5, and 6 that correspond to 1376T, 592T, 1311T, 392T, $1388 \mathrm{~A}$, and $95 \mathrm{G}$ respectively. Mutation appeared with additional peak in three patients carrying 1311T. a) Hemizygous, b) Normal, c) Heterozygous, d) Hemizygous, e) Primer extension wild type, f) PE positive control, and g) PE negative control. 


\section{0 \\ $90 \downarrow$ \\ 100 \\ I I I I I I I I I II I I I I I I I I I I I I I I I I I I I \\ I G I C CA A C CACA I C ICC ICC C I G I I C C G I}

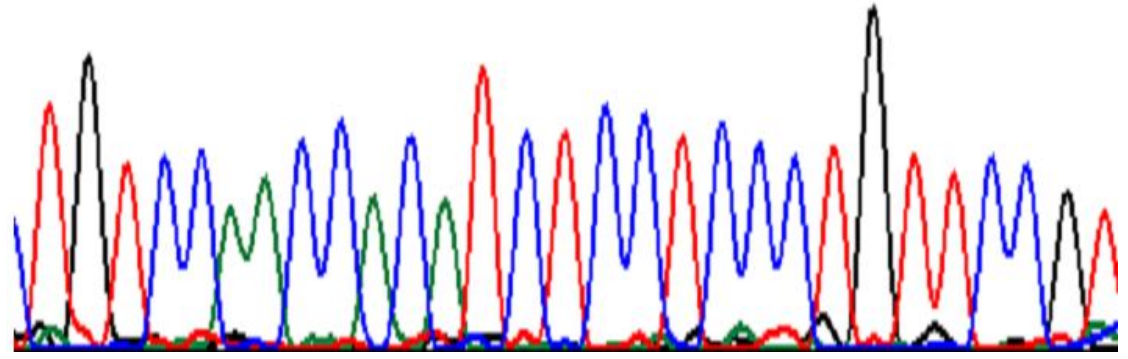

Figure 4: Sequence analysis of the PCR product for exon 6 of the G6PD gene. The arrow indicates $\mathrm{C}$ (cytosine) nucleotide in the normal control for Mediterranean mutation 563C-T.

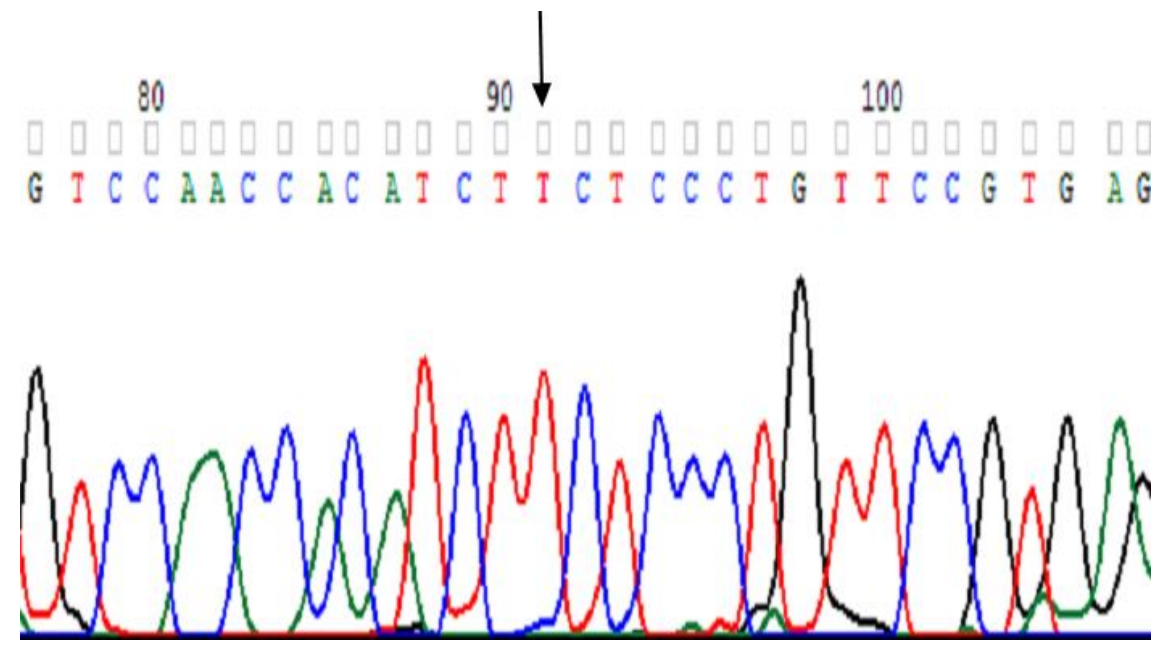

Figure 5: sequence analysis of PCR product for exon 6 with mutantG6PD gene. The arrow indicates $C$ (cytosine) nucleotide substituted by $\mathrm{T}$ (Thymine) in Mediterranean mutation 563C-T leading to change of amino acid serine to phenylalanine at codon 188 characteristic of Mediterranean mutation.

Mediterranean mutations were confirmed by DNA sequencing (Figure 4 and 5).

\section{DISCUSSION}

The inhabitants of the United Arab Emirates are ethnically diverse. UAE has high frequencies of blood disorders including thalassemia, sickle cell disease, and G6PD deficiency [7]. The prevalence of G6PD deficiency in national populations is significantly higher (7.4\%) compared to the non-nationals $(3.8 \%) \quad(p<$ $0.001)$. This reflects high incidence of the disease in the region. Earlier studies on G6PD deficiency among UAE national males living in Al-Ain have reported frequencies of 9.1 to $11 \%$ $[5,6]$ which is slightly higher than the frequency of $7.4 \%$ seen in this study. The frequency of G6PD deficiency in the UAE is less than that in Kuwait (19\%) [8], Bahrain (21\%), and Oman (27\%) [4,9]. Moreover, the variation of G6PD deficiency within different geographical regions in Oman ranges between 8.7 and $29 \%$ [10]. In contrast, the prevalence of G6PD in the UAE population is relatively higher than that in the populations residing in some other Mediterranean countries such as Italy (1 to $2 \%$ ) [11], Spain (1\%) [12], Turkey (1.2\%) [13]. The frequencies of G6PD deficiency which have been reported throughout the Eastern Mediterranean Region ranges from $3.6 \%$ in Jordan to $39.8 \%$ in Eastern Saudi Arabia [14].

In this study, the prevalence of G6PD deficiency is higher in males $(7.2 \%)$ than in females $(2.6 \%)$. In UAE nationals the prevalence among males $(11.6 \%)$ is significantly higher compared to females $(3.6 \%, p<0.001)$. The molecular defects of the G6PD gene have been characterized in 225 subjects in the Abu Dhabi region out of which 144 were UAE nationals and 81 were non-UAE nationals. Five variants have 
been identified. The three most prevalent types of G6PD mutations (Mediterranean, $A^{-}$, and Aures) in this study represent $99.3 \%$ (142/143) of the cases in the UAE nationals. The other two variants (Union and Chatham) showed lower prevalence rate $1.3 \%(3 / 224)$ with two of them belonging to Philippines which carried the Union variant C1360T.

One patient from the UAE carried the Chatham variant G1003A, which is rarely present in the neighboring Arab countries [15]. The mutation in the remaining one subject has not been identified. The G6PD Mediterranean C563T has been identified as the most common variant among the Abu Dhabi populations. The high gene frequency of the G6PD Mediterranean variant among the Abu Dhabi UAE national population agrees with previous findings which show that it is the most common G6PD deficient variant in South East Asia and the Mediterranean region [16].

In this work, silent polymorphisms, $\mathrm{C} / \mathrm{T}$ at nucleotide 1311 (p.Tyr437Tyr) was found in 170 of 173 cases of Mediterranean mutation which shows strong association between these two mutations. This silent mutation has been detected via the DHPLC of exon 11 of the G6PD gene in this study. The second most frequent variant detected in the current study was Aures $143 \mathrm{~T} \rightarrow \mathrm{C}$ found in $12 \%$ of cases of G6PD deficiency, 17 (7.5\%) were UAE nationals, 9 (4\%) Yemeni and $1(0.4 \%)$ was Omani national. Molecular studies have confirmed that the Aures variants in the UAE nationals are similar to those described in western Saudi Arabia with 6 out of $49(12.3 \%)$ deficient Saudi subjects [17].

A previous study carried out in Jeddah, a province of Saudi Arabia also reported the prevalence of Aures variant (10\%), where in 11 out of 110 G6PD deficiencies. However, it has been found earlier in the central part of Saudi Arabia (Riyadh region) that 7 out of 20 (35\%) are deficient, which is lower than the previous findings reported [18].

The data of the current study have shown that the G6PD A- variant, which is predominant in Africa, was also detected in 21 out of 224 (9.4\%) cases among the total number of the G6PDdeficient subjects. Out of these $8.3 \%$ were found among the G6PD-deficient subjects of the UAE nationality.

The G6PD A- variant has been reported mainly in Africa, the Mediterranean region and the Middle East where the G6PD A- variant accounts for about 90 or even 100\% of G6PD deficiency, such as that in Nigeria [19].

This study is the first report to document the presence of Chatham mutation among UAE citizens with a frequency of $0.7 \%$ (1 of 143); the highest prevalence of this mutation was found in Northern Iran (27\%) [20]. The presence of this mutation in UAE might have been occurred due to the gene transferred from neighboring countries such as Iran to the Gulf countries.

\section{CONCLUSION}

The prevalence of G6PD deficiency among UAE nationals in Abu Dhabi is $7.4 \%$ which is lower than in other Gulf countries. The molecular basis of G6PD deficiency in the current study within UAE nationals reveals prevalence of G6PD deficiency Mediterranean mutation and Aures mutation, which are close to values reported in other studies for Arab countries such as Jeddah in Saudi Arabia and Kuwait. For the Aures mutation, it has been documented as a specific mutation for the central and southern parts of the Arabian Peninsula. Thus, the findings of the present study may suggest that UAE nationals might have descended from authentic Arab tribes. The nucleotide $1311 \mathrm{~T}$ is a frequent polymorphism observed in the Abu Dhabi District. Further studies are warranted to investigate the new unidentified G6PD mutation in this study.

\section{Limitations of the study}

This study has been done only in one hospital (Mafraq Hospital), and does not reflect the whole picture of G6PD prevalence in Abu Dhabi district. It is highly recommended that a comprehensive study that includes all hospitals and medical centres in the district should be performed to better determine the accurate prevalence rate of G6PD Mediterranean mutation among children (3 -6 years).

\section{COMPETING INTERESTS}

The authors declare that there is no conflict of interest.

\section{ACKNOWLEDGMENT}

The authors would like to extend their sincere thanks to the administration of The Zayed Bin Sultan Al Nahayan Charitable and Humanitarian Foundation, Abu Dhabi, United Arab Emirates and Universiti Sains Malaysia for their support and provision of facilities for the execution of this work. 


\section{REFERENCES}

1 Sulaiman AM, Saghir SAM, Al-Hassan FM, Yusoff $N M$ Zaki AH. Molecular Characterization of Glucose-6Phosphate Dehydrogenase Deficiency in a University Community in Malaysia. Trop J Pharm Res 2013; 12: 363-367.

2 Haidar B, Mason P, Berrebi A, Ankara-Badu G, Al-Ali A, Oppenheim A, Luzzatto L. Origin and spread of the G6PD variant (G6PDMediterranean) in the Middle East. Am J Hum Genet 1990; 47: 10131019.

3 Anvery SM. Glucose-6-phosphate dehydrogenase deficiency in Abu Dhabi. Emirates Med J 1980; 1 : 24-26.

4 Daar S, Vulliamy T, Kaeda J, Mason PJ, Luzzatto L. Molecular characterization of G6PD deficiency in Oman. Hum Hered1996; 46 (3): 172-176.

5 Bayoumi RA, Nur-E-Kamal M, Tadayyon M, Mohamed K, Mahboob B, Qureshi M M, Lakhani MS, Awaad MO, Kaeda J, Vulliamy TJ, Luzzatto L. Molecular characterization of erythrocyte glucose-6phosphate dehydrogenase deficiency in Al-Ain district, United Arab Emirates. Hum Hered 1996; 46:136-141.

6 Abdulrazzaq YM, Micallef $R$, Qureshi MM, Dawodu A, Ahmed I, Khidr A, Bastaki SMA, Al-Khayat Al, Bayoumi RA. Diversity in expression of glucose-6phosphate dehydrogenase deficiency in females. Clin Genet 1999; 55(1): 13-19.

7 Al-Gazali L, Ali BR. Mutations of a Country: A Mutation Review of Single Gene Disorders in the United Arab Emirates (UAE). Human Mutation 2010; 31(5): 505-20.

8 Poggi V, Town M, Foulkes N, Luzzatto L. Identification of a single base change in anew human mutant glucose-6-phosphate dehydrogenase gene by polymerase-chain-reaction amplification of the entire coding region from genomic DNA. Biochem J 1990; 217(1): 157-160.

9 Shaker $Y$, Onsi A, Aziz R. The frequency of glucose-6 phosphate dehydrogenase deficiency in newborns and adults in Kuwait. Am J Hum Genet 1966; 18: 609-613.
10 White J, Ghristie B, Nam D, Daar S, Higgs D. Frequency and clinical significance of erythrocyte genetic abnormalities in Omanis. J Med Genet 1993; 30: 396-400.

11 Smith $M$. The incidence of G6PD deficiency in a population of Greek, Italian and Yugoslav origin in Australia. Med J Aust 1976; 25: 485-486.

12 Cladera SA, Oliva BE, Torrent QM, Bartolozzi CE. Prevalence of glucose -6- phosphate dehydrogenase deficiency in a student population on the island of menorca. Sangre (Barc), 1997, 42: 363-367.

13 Keskin N, Ozdes I, Keskin A, Acikbas I, Bagci $H$. Incidence and molecular analysis of G6PD deficiency in the province of Denizli, Turkey. Med Sci Monit 2002; 8: 453-456.

14 Beutler E. The molecular biology of G6PD variants and other red cell enzyme defects. Ann Rev Med 1992; 43: 47-59.

15 Al-Fadhli S, Kaaba S, Elshafey A, Salim M, Al-Awadi $A$, Bastaki L. Molecular characterization of glucose-6-phosphate dehydrogenase gene defect in the Kuwaiti population. Arch Pathol Lab Med 2005; 129 (9): 1144-1147.

16 Beutler E. Study of glucose-6-phosphate dehydrogenase: history and molecular biology. Am J Hematol 1993; 42: 53-58.

17 Al-Jaouni SK, Jummanah Jarullah EA, Moradkhani K. Molecular characterization of glucose-6phosphate dehydrogenase deficiency in Jeddah, Kingdom of Saudi Arabia. BMC Res Notes 2011; 4 (1): 436.

18 Niazi G, Adeyokunu A, Westwood B, Beutler E. G6PD Aures: a rare mutant of G6PD in Saudi Arabia: molecular and clinical presentations. Saudi Med J 1996; 17: 311-314.

19 Ademowo OG, Falusi AG. Molecular epidemiology and activity of erythrocyte G6PD variants in a homogeneous Nigerian population. East African Med J 2002; 79: 42-44.

20 Mesbah-Namin SA, Sanati MH, Mowjoodi AR, Mason PJ, Vulliamy TJ, Noori DM. R. Three major G6PD deficient polymorphic variants identified in Mazandaran state of Iran. Brit J Haemat 2002; 117: 763-764. 